\title{
Aerobic exercise and octopamine protect against deep-frying oil-induced cardiomyocyte apoptosis through modulation of caspase and pro-caspase 3
}

\author{
Pantea kianmehr \\ Islamic Azad University \\ Maghsoud Peeri \\ Islamic Azad University \\ Parvin Farzanegi \\ Islamic Azad University
}

Mohammad Ali Azarbayjani ( $D$ m_azarbayjani@iauctb.ac.ir)

Islamic Azad University https://orcid.org/0000-0002-3502-7487

\section{Research}

Keywords: Cardiomyocytes, Aerobic exercise, Apoptosis, Octopamine, Deep-frying oil

Posted Date: September 26th, 2020

DOI: https://doi.org/10.21203/rs.3.rs-16706/v4

License: (a) (i) This work is licensed under a Creative Commons Attribution 4.0 International License.

Read Full License 


\section{Abstract}

Background: Deep-frying is a common cooking method accompanied by production of carcinogenesis substance such as acrolein. Acrolein is a toxic byproduct of lipid peroxidation that is involved in the development of pulmonary, cardiac, and neurodegenerative diseases. This study aimed to explore the effect of aerobic exercise (EXE) and octopamine (OCT) on caspase 3 and pro-caspase 3 expression levels in the heart tissue of rats were fed deep-frying oil (DFO).

Methods: 30 male Wistar rats were divided into 5 groups ( $n=6$ in each) including 1 ) control (CO), 2) DFO, 3) DFO+EXE, 4) DFO+OCT and 5) DFO+EXE+OCT. The apoptotic effects of DFO on heart and cardiomyocytes' fibers were examined by TUNEL assay and Masson's trichrome staining respectively. Also caspase 3 and pro-caspase 3 genes and proteins expression in all groups were evaluated using quantitative real-time PCR and western blotting method, respectively.

Results: Data showed a significant increase in apoptotic cells in the DFO group $(P<0.05)$. Masson's trichrome stain analysis demonstrated that the number of cardiomyocytes' fibers are decreased, and collagen deposition is increased in the DFO group. In comparison, the collagen percentage was significantly reduced in DFO+EXE, DFO +OCT and DFO+EXE+OCT groups. Also, the expression level of caspase 3 and pro-caspase 3 was significantly decreased in DFO+EXE+OCT group $(P<0.05)$.

Conclusions: The results of this study show that DFO lead to programmed cell death via the activation of caspases in heart tissue. However, it seems that aerobic exercise with octopamine supplementation improves heart tissue through decreasing in the expression of caspase 3 and pro-caspase 3 that inhibit apoptosis.

\section{Background}

The heart muscle is made up of cardiomyocytes. These specialized cells themselves are made of myofibrils that consisting of contractile units included long sarcomere chains. [1, 2]. Cardiovascular disease (CVD) is one the most cause of mortality worldwide. Inefficiency and death of cardiomyocytes are considered as a primary contributing factor to these disease [3].

Although, under normal circumstances, cell death occurs to maintain the body's homeostasis, disruption of this phenomenon leads to pathological conditions [4]. Apoptosis (programmed cell death) is a common kind of death which has been observed in cardiomyocyte in several cardiac diseases such as ischemic heart disease, heart failure, and aging [5].

A direct association between CVD risk and consumption of cooking oil polar compounds has been reported [6]. Deep-frying is a cooking way in which food be submerged in any kind of hot oil [7]. Deepfrying produces desirable or undesirable flavor compounds and alternate the flavor stability and quality of the oil through oil hydrolysis, oxidation, and polymerization. It has been also demonstrated that the heating of dietary oils and fats results in lipid oxidation and apoptotic induction. [8]. 
Caspases belongs to proteases family which have critical roles in inflammation and apoptosis [9]. Activation of caspases leads to the onset of pathways resulted in apoptosis.[10]. Among Caspases, Caspase 3 is a frequently activated protease which involved in death cell and specially cleavage some key cellular proteins. [11]. This caspase is inactive and after activation execution phase of apoptosis is started. Various signaling pathways of apoptosis can be different before this event but usually follow the same pathway [12].

Octopamine is a sympathomimetic amine with similar characteristics to norepinephrine. This false neurotransmitter affects adrenergic and dopaminergic systems. It is naturally present in many plants such as oranges and is derived from the synthesis of $D-(-)$ - enantiomer octopamine by $\beta$-hydroxylation of tyramine and the enzyme dopamine $\beta$-hydroxylase. It is marketed under the trade names of Epirenor, Norden and Norfen (14). Octopamine leads to increase the amplitude and frequency of the heartbeat and has been suggested not to be the transmitter of cardio-accelerator neurons [13]. Octopamine was appleyed to control hypotensive disorders and it was showed that doses of $450-600 \mathrm{mg} /$ day increased blood pressure [14]. However, studies about the supplementation of octopamine on heart tissue and myocardicyte apoptosis in cardiovascular disease are limit. It was reported that endurance cycle performance, substrate oxidation or circulating hormonal concentrations were not changes by administration of low dose of octopamine which could be due to the low serum octopamine concentrations [15].

Regular exercise are suggested to help patient with CVD to increase the life quality. It alone or in combination with other factors including supplement or psychological support have been effective [16]. Sujkowski et al (2014) show that octopamine drives endurance exercise adaptations in drosophila [17]. There are limit study on the effects of exercise with octopamine in cardiomyocyte. The aim of this study was to investigate the impact of aerobic exercise and octopamine supplement on cardiomyocytes histopathology and assessing the expression of Caspase 3 and pro-Caspase 3 in heart tissue in DFOtreated rats.

\section{Materials And Methods}

\section{Animals}

In this experimental study, 30 healthy adult male Wistar rats (Rattus norvegicus) with eight weeks old and weighing between 200 to $250 \mathrm{~g}$ were obtained from Pasteur Institute of Iran (Tehran, Iran). The rats were exclusively housed in plastic cages covered by sawdust bedding in the temperature of $20 \pm 2{ }^{\circ} \mathrm{C}$ and humidity about $60 \%$. All rats had access to a standard diet and urban water. The. The lighting period was held at $12 \mathrm{~h}$ light $/ 12 \mathrm{~h}$ dark cycle. All the procedures were approved and monitored by the ethics committee of laboratory animals of Islamic Azad University (Tehran, Iran). The procedures followed the National Institutes of Health guide for the care and use of laboratory animals (NIH Publications No. 8023, revised 1978) (Ethical code: IR.MUK.REC.1398.242). In this study, the animals were randomly assigned to 5 groups $(n=6)$. Groups were as follows: 
1) CO: control (rats were not received any treatments)

2) DFO group: rats were received deep-frying oil

3) DFO+EXE group: rats were received deep-frying oil deep-frying oil, then did aerobic exercise

4) DFO+OCT group: rats were received deep-frying oil deep-frying oil, then were prescribed octopamine.

5) DFO+EXE+OCT group: rats were received deep-frying oil deep-frying oil, then did aerobic exercise and were prescribed octopamine simultaneously.

\section{Chemicals}

\section{Deep-frying oil Preparation}

Deep-frying oil (DFO) was made by frying $5 \mathrm{~kg}$ catfish three times in $2.5 \mathrm{~L}$ cooking palm oil at $200^{\circ} \mathrm{C}$ (measured with a cooking thermometer) for $15 \mathrm{~min}$ for 3-times [18-20]. After every frying, the oil was let to cool at room temperature for 5 hours [21]. For DFO, lipid hydroperoxide content was $90.94(\mathrm{meq} / \mathrm{kg})$, also total fatty acid composition represent in table1. DFO was gavage five days per week $(10 \mathrm{ml} / \mathrm{kg})$ for four weeks.

\section{Octopamine}

OCT (Sigma, UK) was dissolved in distilled water and then diluted with a Krebs-bicarbonate solution. OCT (100 $\mu \mathrm{L}$ in each injection) was injected intraperitoneally five days per week $(10 \mathrm{~mL} / \mathrm{kg})$ for four weeks.

\section{Aerobic exercise protocol}

Rats of EXE group were introduced to treadmill running for a habituation period, during which each animal exercised on a motorized rodent treadmill at $9 \mathrm{~m} / \mathrm{min}$ for $20 \mathrm{~min}$ per day (including $10 \mathrm{~min}$ at a prescribed speed, a 5-min warm-up, and 5-min cool-down). After the habituation period, rats were subjected to run at moderate exercise intensity for five days per week over four weeks (total duration of the exercise protocol and OCT supplementation). On the first day of exercise, the training began with the speed of $11 \mathrm{~m} / \mathrm{min}$ for $10 \mathrm{~min}$ per day. The speed gradually increased to $20 \mathrm{~m} / \mathrm{min}$ over the duration of the experiment. The exercise duration was also increased 2 min per day over the same period until it reached $26 \mathrm{~min} /$ day at the end of the second week.

\section{Animal tissue sampling}

Forty-eight hours after the last training session, all rats were sacrificed and their heart tissue were removed immediately and washed in saline normal $0.9 \%$. The heart tissues (ventricle) were divided into two parts for freezing $\left(-80^{\circ} \mathrm{C}\right)$ and fixing $(10 \%$ neutralized formalin). Bodyweight and heart weight also measure at the end of the protocol (Table 2). 


\section{Terminal transferase dUTP nick end labeling (TUNEL) assay}

The apoptotic effects of aerobic exercise and octopamine on heart tissue samples in DFO treated rats were determined using the transferase-mediated deoxyuridine triphosphate (dUTP)-digoxigenin nick end labeling assay. Samples were fixed with $4 \%(\mathrm{w} / \mathrm{v})$ paraformaldehyde and processed using a commercial kit (In Situ Cell Death Detection Kit; Roche, Germany) in accordance with the manufacturer's instructions. Cells were stained with Hoechst dye and imaged in a fluorescent microscope. The data were expressed as a percentage of the area of terminal deoxynucleotidyl transferase deoxyuridine triphosphate nick end labeling (TUNEL)-positive cells in ten random fields. The number of TUNEL positive cells was measured carefully in 3 sections of the heart tissue per animal. The percentage of positively stained cells in the area was analyzed with Image $J$ software v1.8 (NIH, USA).

\section{Histopathological examination: H\&E and Masson's trichrome staining}

Histopathological examination of cardiac muscle tissue was done using hematoxylin and eosin (H\&E) staining. To perfusion fixation, fresh heart tissues were placed into $10 \%$ formalin and then dehydrated using alcohol. Finally, samples were embedded in paraffin, sectioned, and stained with H\&E (SigmaAldrich, UK). H\&E staining was performed according to the previously described methods [22]. Myofibers were counted in 10 fields of muscle sections for each rat.

Masson's trichrome stain (Histogenotech. Co., Tehran, Iran) were performed according to kit instructions except Aniline Blue-Solution incubation time which was 90 min in this study. The prolonged incubation is a standard procedure of trichrome staining for muscle. Briefly, heart tissue slides deparaffinized by submerging into three series of absolute xylene for $4 \mathrm{~min}$. After that, the slides were washed with running tap water for $2 \mathrm{~min}$. Then, slides were treated with the phosphomolybdic acid solution for another $10 \mathrm{~min}$ as a mordant. Next, slides immediately were submerged into methyl blue (Merck, Germany). Finally, slides were washed in running water for 2 min and treated with $1 \%$ acetic acid solution for 1 min.

\section{RNA extraction}

RNA were extracted from the heart tissue using TRizol reagent (Invitrogen, USA) according to the manufacturer's instruction. The quality of the extracted RNA was determined based on the 260/280 absorbance ratio, measured by the NanoDrop spectrometer (Thermo Scientific, USA).

\section{cDNA synthesis and quantitative RT-PCR analysis (qRT-PCR)}

Synthesis of CDNA was carried out using a universal CDNA synthesis kit (Exiqon, Denmark) according to manufacturer's instructions. Specific primers for Caspase 3 and GAPDH (as housekeeping gene) genes were designed using Primer Premier 5 software (Premier Bio-Soft International, Palo Alto, CA, USA). Their sequences are presented in Table 3. Real-time quantitative PCR reactions were carried out in triplicate by using standard protocols with an $A B I$ step one system ( $A B I, U S A)$. Briefly, in a total volume of $10 \mu \mathrm{l}, 20$ $\mathrm{ng} / \mu \mathrm{l}$ of cDNAs were added to a master mix comprising $10 \mathrm{pmol} / \mu \mathrm{l}$ of each primer (Caspase 3) and $5 \mathrm{~mL}$ of SYBR premix ExTaq II (Thermo Scientific, USA). The program for the run was set as follows: $95^{\circ} \mathrm{C}$ for 
15 min followed by 40 cycles of $95^{\circ} \mathrm{C}$ for $15 \mathrm{~s}, 60^{\circ} \mathrm{C}$ for $30 \mathrm{~s}$, and $72{ }^{\circ} \mathrm{C}$ for $30 \mathrm{~s}$. The PCR reaction was followed by a melting curve program $\left(60-95^{\circ} \mathrm{C}\right.$ with a temperature transition rate of $1{ }^{\circ} \mathrm{C} \mathrm{s}^{-1}$ and a continuous fluorescence reading). GAPDH was quantified as the reference to normalize differences in total RNA levels. The calculation was performed as follows:

Ratio $=\frac{2^{\left.\Delta \mathrm{c}_{\mathrm{T}}, \mathrm{t} \arg \text { et (calibrator-test }\right)}}{2^{\left.\Delta \mathrm{c}_{\mathrm{T}}, \text { ref (calibrator-test }\right)}}$

\section{Western blotting analysis}

Heart tissue was lysed in lysis buffer (RIPA, Beyotime Institute of Biotechnology) supplemented with protease inhibitors (PMSF, Aladdin). The equal amounts of protein were separated by SDS-PAGE with $5 \%-12 \%$ Tris-Glycine gel (Invitrogen) and subjected to standard western blotting analysis. Western blotting was performed using Caspase 3 and pro-Caspase 3 antibodies (1:1000 dilution; Abcam) followed by treating with the corresponding secondary HRP-conjugated antibodies (1:2000 dilution; Abcam, USA). Finally, blots were developed using the ECL western blotting detection system (Amersham Life Sciences Inc., Arlington Heights, IL). The results were subjected to densitometry analysis using the ImageJ software in a blind analysis manner. To ensure that the same amount of proteins was loaded, the GAPDH protein was employed as an internal control.

\section{Statistical analysis}

The statistical analysis of obtained data was performed using the Graph Pad Prism statistical software version 5.01 (GraphPad, San Diego, CA, USA). The normality of the data was evaluated using the Kolmogorov-Smirnov test, two-way ANOVA. Tukey post-Hoc test was used to analyze the data from different groups. Data are expressed as the mean \pm SD. The level of significance was set at $P<0.05$.

\section{Results}

\section{Histopathological H\&E and fibrosis assessment (Masson's trichrome staining)}

Results of the H\&E study showed that cardiac tissue of heart in control and DFO+EXE+OCT groups were cohesive compared to other experimental groups (Fig. 1). The number of myofibrils in Co, DFO+EXE, DFO+OCT and DFO+EXE+OCT groups were significantly more than that $(P \leq 0.01)$ compared to DFO group. Also, the results showed that the diameter of myofibrils in DFO+EXE+OCT group was significantly higher in DFO group in comparison to other groups $(P \otimes 0.031)$.

Masson's trichrome staining results are presented in fig. 2.a. It was observed that amount of collagen fiber in the DFO group increased significantly $(P=0.003)$ compare to the control group. But aerobic 
exercise significantly reduced collagen deposition in DFO+EXE group $(F=38.10, P=0.001, \nabla=0.826)$. Also, octopamine treatment was able to reduce collagen levels in heart tissue significantly $(F=27.62, P=$ $0.001, \nabla=0.775$ ). As well, DFO+EXE+OCT group had significantly reduced number of collagen fibers in

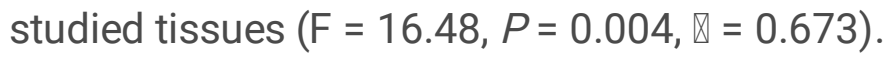

\section{Apoptosis}

As shown in Figure $3 a$ and $b$, the number of apoptotic cells in the tissue was significantly higher in all DFO groups $(P<0.05)$. Aerobic exercise and octopamine treatment significantly reduced the number of

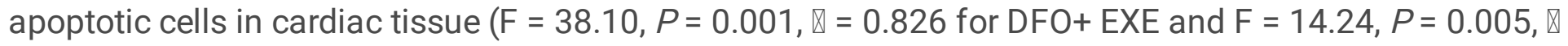
$=0.640$ for DFO+OCT groups). Also simultaneous regime of aerobic exercise and octopamine treatment

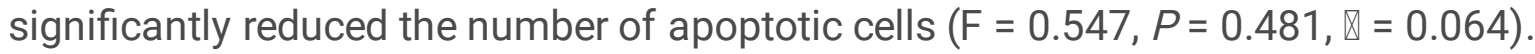

\section{Caspase 3 gene expression}

Analysis of gene expression revealed that DFO resulted in a significant increase in expression of Caspase-3 $(P=0.001)$ in heart tissue. However aerobic exercise and octopamine treatment alone or in combination significantly reduced the expression of Caspase 3 in cardiac tissue $(F=9.16, P=0.007, \nabla=$ 0.375 for DFO+ EXE and $F=16.39, P=0.001, \nabla=0.506$ for DFO+ OCT groups). This reduction was higher in the case of combination of aerobic exercise and octopamine $(F=0.003, P=0.954, \square=0.003$ for DFO+ EXE+OCT) results has been presented in fig. 4 .

\section{Caspase 3 and pro-Caspase 3 proteins expression}

Results of Western blot are depicted in Fig. 5A. The levels of both Caspase 3 and pro-Caspase 3 proteins increased significantly in the heart tissue of DFO group $(P=0.001)$. However aerobic exercise and octopamine treatment alone or in combination significantly reduced the level of Caspase 3 protein in cardiac tissue (for DFO+ EXE+OCT: $F=19.32, P=0.002, \nabla=0.707$ ). On the other hand, aerobic exercise did not have a significant effect on the expression of Pro-Caspase 3 protein $(F=0.903, P=0.370, \nabla=$ 0.101). But octopamine reduced the level of this protein $(F=14.19, P=0.005, \mathbb{Q}=0.651)$. The ratio of

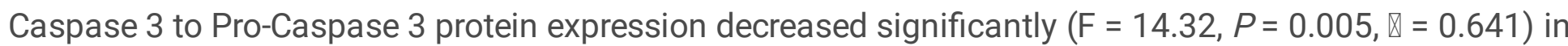
DFO+ EXE, DFO +OCT and DFO+ EXE+OCT groups (Fig. 5 B and C).

\section{Discussion}

Due to the importance of cooking and the taste of food, various cooking techniques have been proposed all over the world [23]. In some countries, one of the welcomed methods is frying food at a high temperatures in heated oils. This process makes the food taste special, crispy and delicious [24]. However, despite the good taste of this type of food, it can have detrimental effects on human health. Cardiovascular system highly affected by high-fat diets. DFO due to the disruption in their fat structure can have worst effect than a high fat regime [25]. Doing exercise or using herbal and pharmacological supplements decrease or delay incidence of these adverse effects [26]. This study shows that exposure to 
DFO for four weeks disrupted cardiomyocyts' regularity and increased collagen deposition as well as significant increase of apoptotic cells in heart tissue. The results also consists of the upregulation of caspase 3 and pro-caspase 3.

It seems that one of the reasons of cardiomyocyte destructions by DFO is the structural changes of oil because of boiling and frying $[24,27]$. It has been show that fried fish oil associated with reduced ejection fraction, lower cardiac output and higher systemic vascular resistance in older adults[28]. Various chemical reactions occur during the frying process such as thermal oxidation, hydrolysis, and polymerization as a result of high temperatures in the presence of air and moisture. One of structural changes is change the fatty acid configuration from the CIS isomer to the Trans isomer after oil repeated heating at high temperatures $\left(180^{\circ} \mathrm{C}\right)$ [29]. This configuration change causes polyunsaturated fatty acids to exhibit the undesirable properties observed in the use of saturated fatty acids including increased serum cholesterol levels and higher low-density lipoprotein (LDL) cholesterol in particular [30].

Also, during the frying process, natural antioxidants are reduced, so, after repeated heating, antioxidants can no longer prevent the oxidation of fatty acids of the oil [31,32]. This process increase the reactive oxygen spice (ROS) in cardiomyocytes which can be a trigger to start apoptosis pathway [33]. In this study although the oxidative stress marker was not measured, increased in level of apoptosis markers (caspase 3 and pro-caspase 3) was determined in heart rats which received DFO for four weeks. Gupta et al. evaluated the effect of different deep fried vegetable oil on the cardiovascular system in rats model and showed that deep-fried vegetable oil increased plasma nitric oxide level and caused blood pressure and aortic structure changes [34]. Compounds formed in DFO during oil drying are able to cause DNA adducts and to increase chromosomal aberrations such as breaks, deletion, exchanges and micronuclei. If normal repair processes failed in cells carrying these abnormalities, the cells were driven to apoptosis [35]. Also the histopatilogical studies revealed that the diameter of muscle fibers in DFO group was significantly less than that of other groups.

We found that four weeks of aerobic exercise and octopamine supplementation improve heart tissue integration and downregulated caspase and pro-caspase 3 in the cardiac cell. Regular aerobic exercise can increase the antioxidant enzymes in various tissues, especially in the heart [36,37]. This statement is in accordance with studies that reported antioxidant defense increase and fat and protein peroxidation reduction. So, it is a reinforcing antioxidant defenses way to improve the neutralization of free radicals and reduce oxidative stress. [38,39]. In this study, decreasing in the levels of apoptotic cells indicated control of apoptotic enzymes which can possibly be due to the reduction of oxidative stress as a result of exercise. So the measurement of antioxidant capacity of cardiac tissue was recommended for future studies.

Apart from mentioned mechanism, it has been shown that cellular adaptation to exercise can be associated with both endogenous and exogenous factors [40]. Exercise induces cardiac growth via hypertrophy and renewal of cardiomyocyte [41]. Also, it is stated that exercise induces endothelial progenitor cells to proliferate, migrate and differentiate into mature endothelial cells, giving rise to 
endothelial regeneration and angiogenesis in heart tissue [42]. All of these events improve apoptosis in heart.

In this study, rats received OCT showed better histopatological results and had less apoptotic enzymes in comparison to DFO groups. Studies demonstrated the ability of octopamine to activate B3 adrenoreceptors and stimulate lipolysis [43], suggesting octopamine could influence fat metabolism[44]. Therefore OCT supplements with lipolysis-stimulating activity, acts as an efficient anti-obesity. OCT with controlling harmful fats caused by DFO has a role to reduce the cellular damages and apoptosis. In previous study on octopamine, we showed that OCT supplementation significantly reduced NF-KB and TNF-a gene expression levels in the heart tissue of rats fed DFO [27]. Therefore, it seems that one of the possible mechanisms of action of octopamine in heart improvement is the reduction of the ROS, which leads to a reduction in inflammation and oxidative stress and, ultimately, apoptosis. Clinical evidence suggests inflammation and oxidative stress linked to the overgeneration of ROS [45].

Combination of aerobic exercise and octopamine consumption had downregulating effect on both studied genes (Caspase and pro-Caspase 3). So overcoming to the cytotoxicity of DFO (reduce apoptosis induced by DFO) is expected. Antioxidant activity of exercise in association with lipolysis activity of OCT accelerated good effect of each of them lonely.

\section{Study strengths and limitations}

This study provided a detailed base data regarding to myocardial injury and apoptosis markers in protein and gene levels. However, as a limitation we did not characterize DFO and we did not measure lipid peroxidation acid value, peroxide value, smoke point, and ultraviolet absorbance at $232 \mathrm{~nm}$. Another limitations of this study is the lack of the investigation the protective effects of exercise and octapamin via assessing the regulation of stress proteins such as MnSOD, ERK, IGF-1 / Akt, HSPs and NF-kB. Also, we did not track the weight gain (especially fat mass) throughout the intervention. Due to the effect of over weighting on cardiovascular system, it remains possible that the impact of the intervention (s) was the result of differences in the weight.

\section{Conclusion}

Based on the results of this study, DFO have detrimental effects on heart tissue through increase cell apoptosis which in a long time can consequence to heart failure and even death. However aerobic exercise training with octopamine can inhibit apoptosis factors in rats exposed to DFO. It seems that performing exercise training with OCT supplementation can improve cardiomyocyte homeostatic disturbances due to apoptosis-induced by DFO. However, more studies (in-vitro and in-vivo) are needed, especially on the human sample to find a life style including both exercise and using supplementary to diminish unwanted outcome of special diets.

\section{Abbreviations}


DFO: Deep-frying oil, CVD: cardio vascular disease.

\section{Declarations}

Ethics approval and consent to participate

The protocol was approved by the Ethics Committee of Islamic Azad University, Tehran, Iran (Code: IR.MUK.REC.1398.242).

Consent for publication

'Not applicable'.

Availability of data and materials

Not applicable.

Competing interests

The authors declare that they have no competing interests.

Funding

No funding

Authors' contributions

All author equally contributes in preparation of this manuscript.

\section{Acknowledgements}

There were no sources of external funding

\section{References}

1. Seifter J, Sloane D, Ratner A: Concepts in medical physiology. Lippincott Williams \& Wilkins; 2005.

2. Kuehnel W: Color Atlas of Cytology, Histology and Microscopic Anatomy. Georg Thieme Verlag; 2003.

3. Reeve JL, Duffy AM, O'Brien T, Samali A: Don't lose heart-therapeutic value of apoptosis prevention in the treatment of cardiovascular disease. Journal of cellular and molecular medicine 2005, 9:609-622.

4. Favaloro B, Allocati N, Graziano V, Di llio C, De Laurenzi V: Role of apoptosis in disease. Aging (Albany NY) 2012, 4:330.

5. Borutaite V, Brown GC: Mitochondria in apoptosis of ischemic heart. FEBS letters 2003, 541:1-5.

6. Ng C-Y, Leong X-F, Masbah N, Adam SK, Kamisah Y, Jaarin K: Heated vegetable oils and cardiovascular disease risk factors. Vascular Pharmacology 2014, 61:1-9. 
7. Thornes N: Food preparation and cooking: cookery units. Student Guide Nelson Thomas Ltd: United Kingdom 1996.

8. Choe E, Min D: Chemistry of deep-fat frying oils. Journal of food science 2007, 72:R77-R86.

9. Mcllwain DR, Berger T, Mak TW: Caspase functions in cell death and disease. Cold Spring Harbor perspectives in biology 2015, 7.

10. Xanthoudakis S, Roy S, Rasper D, Hennessey T, Aubin Y, Cassady R, Tawa P, Ruel R, Rosen A, Nicholson DW: Hsp60 accelerates the maturation of pro-caspase-3 by upstream activator proteases during apoptosis. The EMBO journal 1999, 18:2049-2056.

11. Porter AG, Jänicke RU: Emerging roles of caspase-3 in apoptosis. Cell death and differentiation 1999, 6:99.

12. Samali A, Cai J, Zhivotovsky B, Jones DP, Orrenius S: Presence of a pre-apoptotic complex of procaspase-3, Hsp60 and Hsp10 in the mitochondrial fraction of Jurkat cells. The EMBO journal 1999, 18:2040-2048.

13. Florey $E$, Rathmayer M: The effects of octopamine and other amines on the heart and on neuromuscular transmission in decapod crustaceans: further evidence for a role as neurohormone. Comparative Biochemistry and Physiology Part C: Comparative Pharmacology 1978, 61:229-237.

14. Kuske F: Treatment of hypotensive regulation disturbances by Norphen. Therapie der Gegenwart 1969, 108:1460.

15. Beaumont RE, Cordery P, James LJ, Watson P: Supplementation with a low-dose of octopamine does not influence endurance cycling performance in recreationally active men. Journal of Science and Medicine in Sport 2017, 20:952-956.

16. Anderson L, Oldridge N, Thompson DR, Zwisler A-D, Rees K, Martin N, Taylor RS: Exercise-based cardiac rehabilitation for coronary heart disease: Cochrane systematic review and meta-analysis. Journal of the American College of Cardiology 2016, 67:1-12.

17. Sujkowski A, Ramesh D, Brockmann A, Wessells R: Octopamine drives endurance exercise adaptations in Drosophila. Cell reports 2017, 21:1809-1823.

18. Weber J, Bochi VC, Ribeiro CP, Victório AdM, Emanuelli T: Effect of different cooking methods on the oxidation, proximate and fatty acid composition of silver catfish (Rhamdia quelen) fillets. Food Chemistry 2008, 106:140-146.

19. Limpisathian P, Potasin N, Tinwong C: Processing of durian french fried-type product. In International Workshop on Tropical and Subtropical Fruits 787. 2007: 391-394.

20. Chen $Y$, Yang $Y$, Nie S, Yang X, Wang Y, Yang M, Li C, Xie M: The analysis of trans fatty acid profiles in deep frying palm oil and chicken fillets with an improved gas chromatography method. Food control 2014, 44:191-197.

21. Kianmehr P, Azarbayjani MA, Peeri M, Farzanegi P: Synergic effects of exercise training and octopamine on peroxisome proliferator-activated receptor-gamma coactivator-1a and uncoupling protein $1 \mathrm{mRNA}$ in heart tissue of rat treated with deep frying oil. Biochemistry and biophysics reports 2020, 22:100735. 
22. Mayer P: Hematoxylin and eosin (H\&E) staining protocol. Mitt Zool Stn Neapel 1896, 12.

23. Provost JJ, Colabroy KL, Kelly BS, Wallert MA: The science of cooking: Understanding the biology and chemistry behind food and cooking. John Wiley \& Sons; 2016.

24. Bouchon P, Dueik V: Frying of Foods. In Fruit Preservation. Springer; 2018: 275-309

25. Sun Y, Liu B, Snetselaar LG, Robinson JG, Wallace RB, Peterson LL, Bao W: Association of fried food consumption with all cause, cardiovascular, and cancer mortality: prospective cohort study. $b m j$ $2019,364$.

26. Imran TF, Joseph J: Herbal Therapies and Micronutrient Supplements in Cardiovascular Disease. Cardiovascular Diseases 2017, 1:129.

27. Jafari Amoli F, Hosseini SA, Azarbayjani MA, Farzanegi Arkhazloo P: The Anti-inflammatory Effects of Aerobic Training and Octopamine Consumption in the Heart Tissue of Rats Fed Deep-Fried Oil. Jorjani Biomedicine Journal 2019, 7:11-19.

28. Mozaffarian D, Gottdiener JS, Siscovick DS: Intake of tuna or other broiled or baked fish versus fried fish and cardiac structure, function, and hemodynamics. The American journal of cardiology 2006, 97:216-222.

29. Jung MY, Choi D-S: Protective effect of gallic acid on the thermal oxidation of corn and soybean oils during high temperature heating. Food science and biotechnology 2016, 25:1577-1582.

30. Mensink RP, Katan MB: Effect of dietary trans fatty acids on high-density and low-density lipoprotein cholesterol levels in healthy subjects. New England Journal of Medicine 1990, 323:439-445.

31. Zaghi AN, Barbalho SM, Guiguer EL, Otoboni AM: Frying process: From conventional to air frying technology. Food Reviews International 2019, 35:763-777.

32. Liu Y, Li J, Cheng Y, Liu Y: Effect of frying oils' fatty acid profile on quality, free radical and volatiles over deep-frying process: A comparative study using chemometrics. LWT 2019, 101:331-341.

33. Yang Y-C, Tsai C-Y, Chen C-L, Kuo C-H, Hou C-W, Cheng S-Y, Aneja R, Huang C-Y, Kuo W-W: Pkcס activation is involved in ROS-mediated mitochondrial dysfunction and apoptosis in cardiomyocytes Exposed to advanced glycation end products (ages). Aging and disease 2018, 9:647.

34. Gupta R, Vind SK, Singh SP, Kumar S, Kumar M: The effect of different deep fried vegetable oil on cardiovascular system in rats model. World Journal of Pharmaceutical Research 2014, 3:1130-1139.

35. Ganesan K, Sukalingam K, Xu B: Impact of consumption of repeatedly heated cooking oils on the incidence of various cancers-A critical review. Critical reviews in food science and nutrition 2019, 59:488-505.

36. Wilson D, Johnson P: Exercise modulates antioxidant enzyme gene expression in rat myocardium and liver. Journal of applied physiology 2000, 88:1791-1796.

37. Mochizuki C, Adachi K, Tanaka Y, Seki R: The effect of prenatal moderate aerobic exercise to inhibit the increase of oxidative stress. The Journal of Japan Academy of Health Sciences 2019, 22:5-15.

38. Bouzid MA, Filaire E, Matran R, Robin S, Fabre C: Lifelong voluntary exercise modulates age-related changes in oxidative stress. International journal of sports medicine 2018, 40:21-28. 
39. Simioni C, Zauli G, Martelli AM, Vitale M, Sacchetti G, Gonelli A, Neri LM: Oxidative stress: role of physical exercise and antioxidant nutraceuticals in adulthood and aging. Oncotarget 2018, 9:17181.

40. Moustogiannis A, Theos A: how exercise benefits your health the cellular and molecular mechanisms. Journal of Orthopedic Research and Therapy 2019.

41. Tao L, Bei Y, Zhang H, Xiao J, Li X: Exercise for the heart: signaling pathways. Oncotarget 2015, 6:20773.

42. Bellafiore M, Battaglia G, Bianco A, Palma A: Expression pattern of angiogenic factors in healthy heart in response to physical exercise intensity. Frontiers in physiology 2019, 10.

43. Carpéné C, Galitzky J, Fontana E, Atgié C, Lafontan M, Berlan M: Selective activation of $\beta 3$ adrenoceptors by octopamine: comparative studies in mammalian fat cells. Naunyn-Schmiedeberg's archives of pharmacology 1999, 359:310-321.

44. Tao J, Ma Y-C, Yang Z-S, Zou C-G, Zhang K-Q: Octopamine connects nutrient cues to lipid metabolism upon nutrient deprivation. Science advances 2016, 2:e1501372.

45. Almzaiel AJT: Oxidative stress and inflammation in ischemic heart disease: role of trace elements, oxidants and antioxidants. Journal of Contemporary Medical Sciences 2015, 1.

\section{Tables}

Due to technical limitations, Table 1 is provided in the Supplementary Files section.

Table 2. Bodyweight and heart weight in different groups of study (mean \pm SD)

\begin{tabular}{|lll|}
\hline Heart weight $(\mathrm{g})$ & Body weight $(\mathrm{g})$ & Groups \\
\hline $1.05 \pm 0.03$ & $256 \pm 7$ & Co \\
\hline $1.03 \pm 0.05$ & $265 \pm 4$ & DFO \\
\hline $1.11 \pm 0.07$ & $249 \pm 8$ & DFO+EXE \\
\hline $0.99 \pm 0.03$ & $261 \pm 3$ & DFO+OCT \\
\hline $1.08 \pm 0.07$ & $260 \pm 5$ & DFO+EXE+OCT \\
\hline
\end{tabular}

CO: control (rats were not received any treatments), DFO group: rats were received deep-frying oil, DFO+EXE group: rats were received deep-frying oil deep-frying oil, then did aerobic exercise, DFO+OCT group: rats were received deep-frying oil deep-frying oil, then were prescribed octopamine, DFO+EXE+OCT group: rats were received deep-frying oil deep-frying oil, then did aerobic exercise and were prescribed octopamine simultaneously

Table 3. Primer sequences for real time PCR assessment 


\begin{tabular}{|llll|}
\hline Product length (bp) & TM () & Primer sequences & Gene name \\
\hline 121 & 81.1 & F: 5'- CAT ACT CAG CAC CAG CAT CAC C -3' & GAPDH \\
& & R: 5'- CATACTCAGCACCAGCATCACC -3' & \\
\hline 82 & 80.5 & F:5'- GCACAAAGTGACTGGATGAAC-3' & Caspase 3 \\
& & R:5'- TGTATTCTTACTCTACCGCAC-3' & \\
\hline
\end{tabular}

\section{Figures}
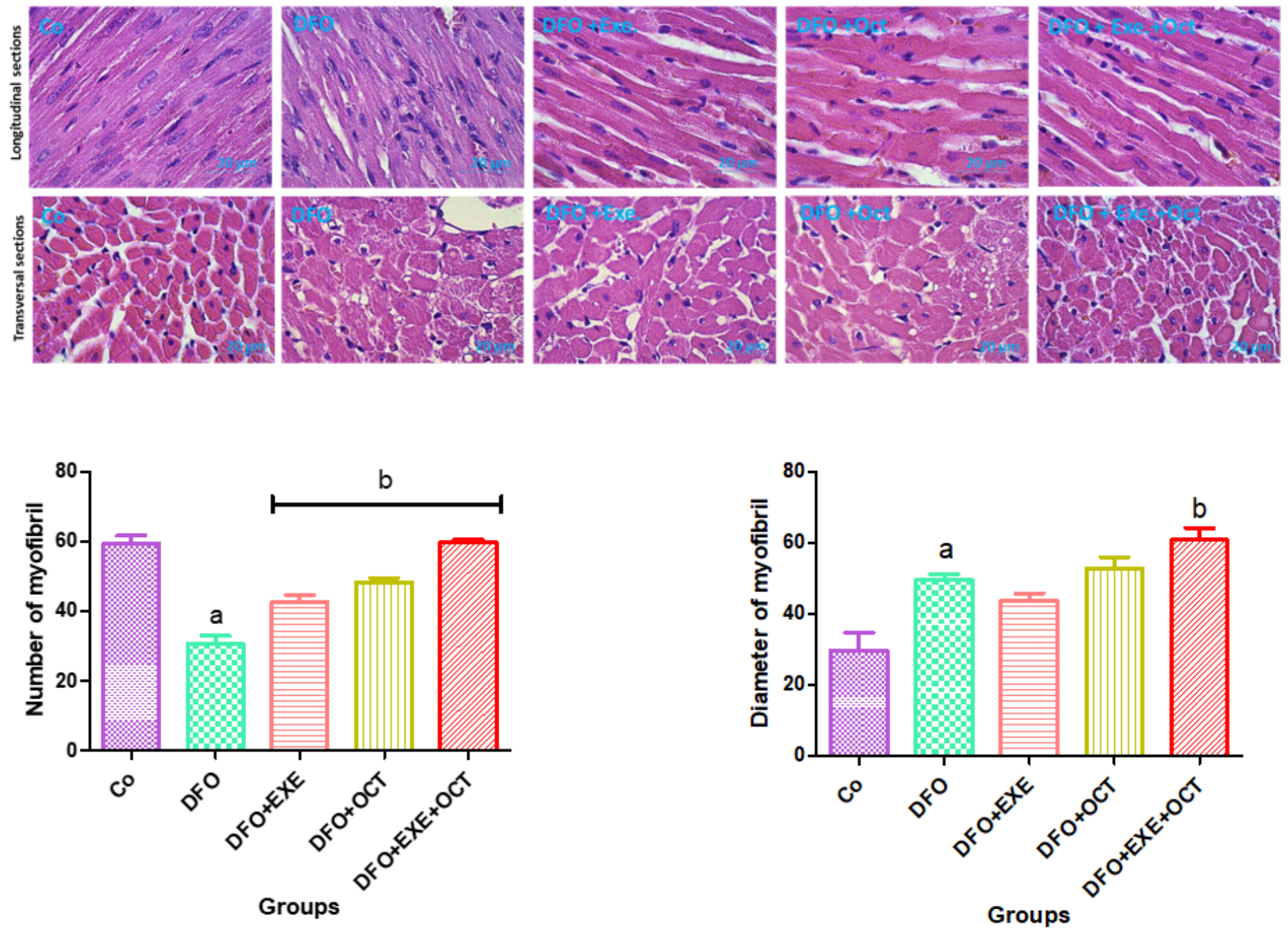

Figure 1

Histopathological examination of cardiac muscle tissue after treatment with DFO in the experimental groups using H\&E staining. Images of longitudinal sections show tissue integrity, and the images of transverse sections show the diameter and number of muscle fibers. a: significant differences with the 
control group at $P \otimes 0.01$, b: significant differences with the DFO group at $P \otimes 0.01$. The results represent the mean \pm SD with five repeats. CO: control (rats were not received any treatments), DFO group: rats were received deep-frying oil, DFO+EXE group: rats were received deep-frying oil deep-frying oil, then did aerobic exercise, DFO+OCT group: rats were received deep-frying oil deep-frying oil, then were prescribed octopamine, DFO+EXE+OCT group: rats were received deep-frying oil deep-frying oil, then did aerobic exercise and were prescribed octopamine simultaneously.
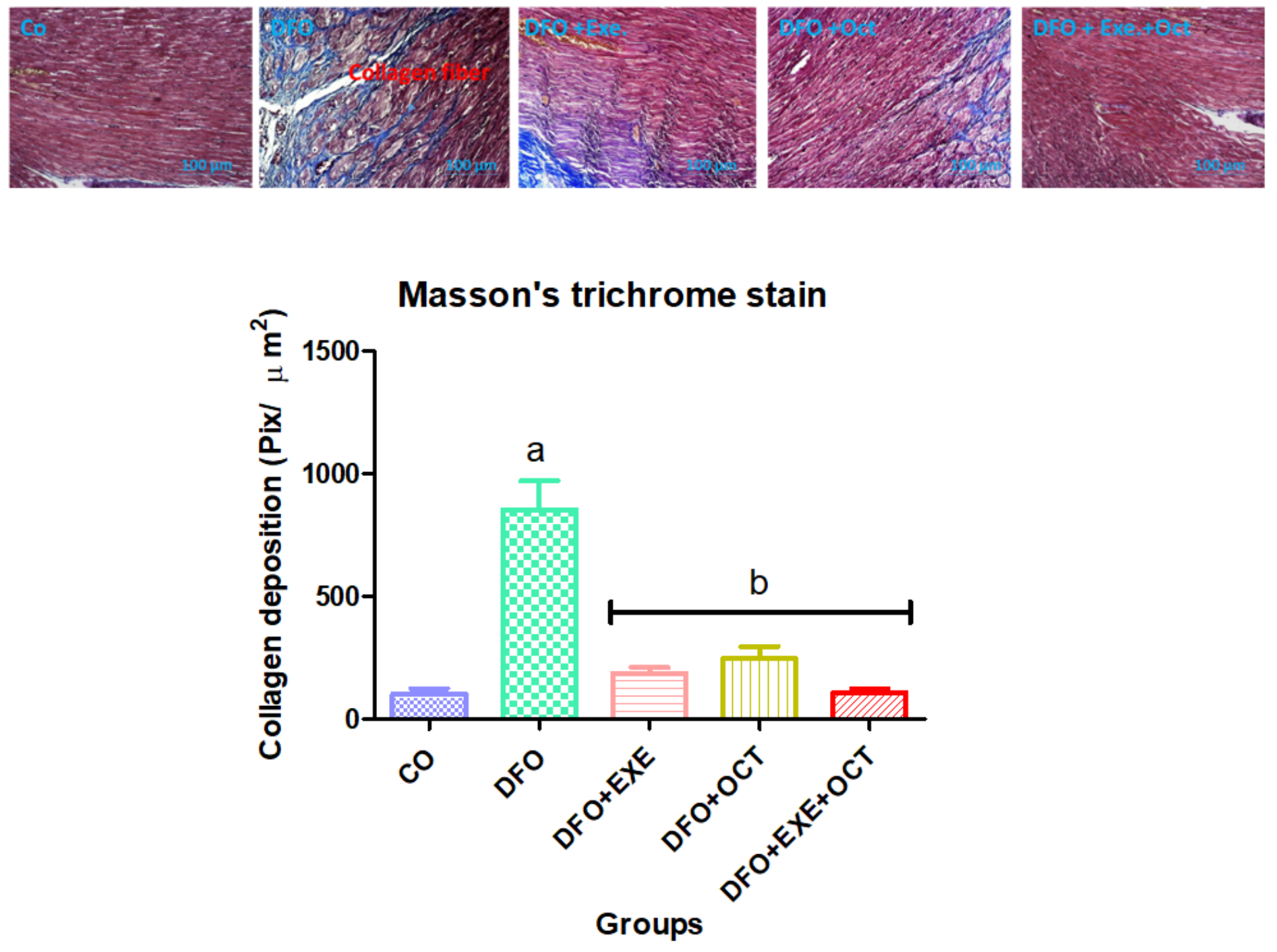

Figure 2

Results of Masson's trichrome staining. Fibrotic tissue and collage deposition were shown in cardiac muscle tissue after treatment with DFO. Blue: fibrous collagen, red: myocytes, black: nuclei a: significantly different with the control group (CO) at $P \otimes 0.0032$, b: significantly different with the DFO at $P \otimes 0.005$. CO: control (rats were not received any treatments), DFO group: rats were received deep-frying oil, DFO+EXE group: rats were received deep-frying oil deep-frying oil, then did aerobic exercise, DFO+OCT group: rats were received deep-frying oil deep-frying oil, then were prescribed octopamine, DFO+EXE+OCT group: rats 
were received deep-frying oil deep-frying oil, then did aerobic exercise and were prescribed octopamine simultaneously. The results represent the mean \pm SD with five repeats.

A)
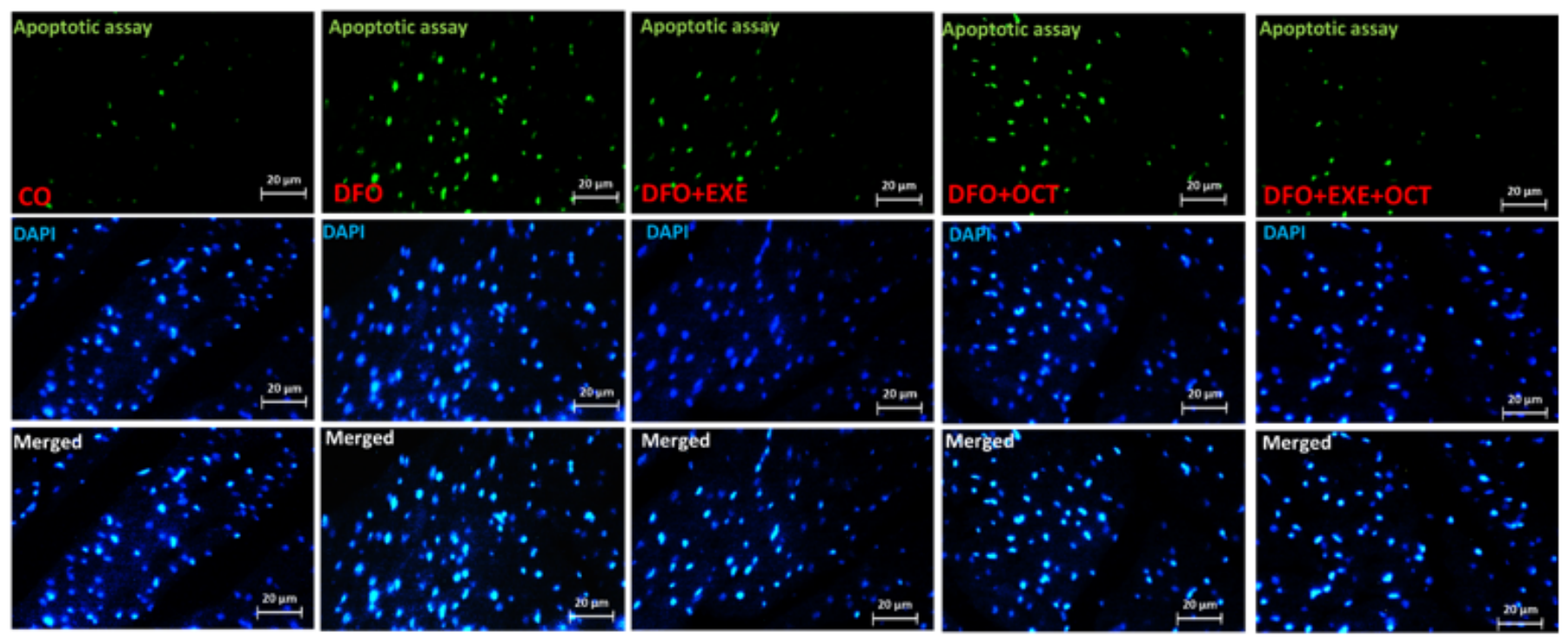

B)

TUNEL assay

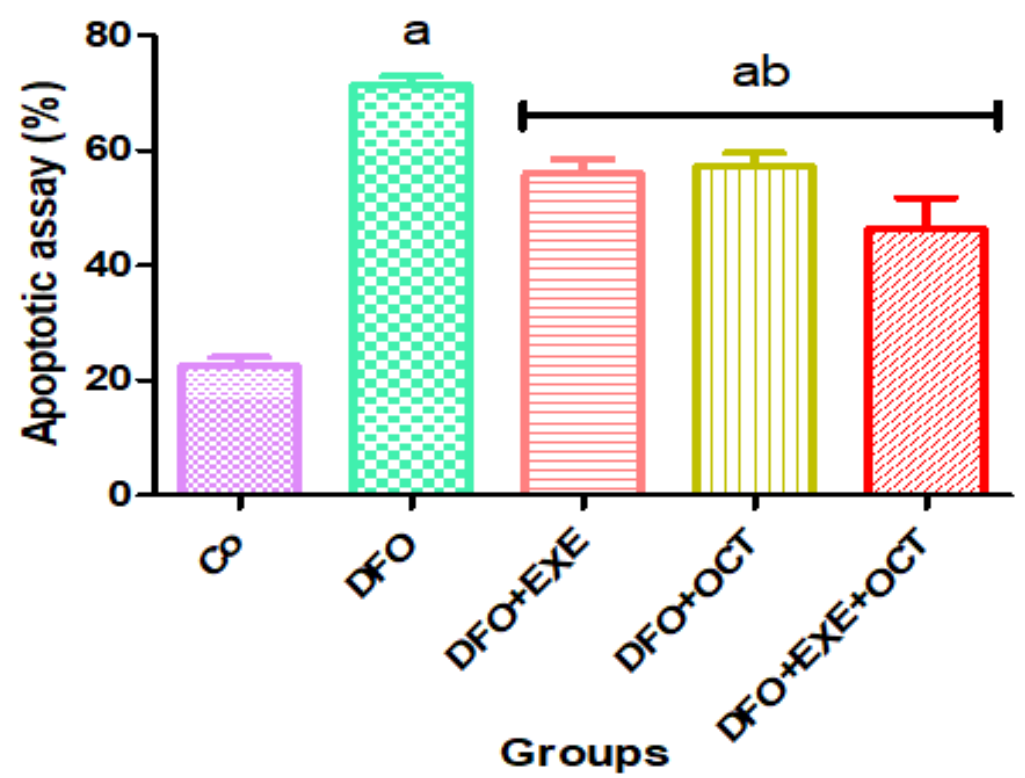

Figure 3

A) Apoptotic effects of DFO were recognized by the TUNEL assay. B) Semi-quantitative analysis of TUNEL assay. a: significantly different from the control group (CO) at $P \otimes 0.001$, b: significantly different from the DFO at $P \otimes 0.0042$. CO: control (rats were not received any treatments), DFO group: rats were 
received deep-frying oil, DFO+EXE group: rats were received deep-frying oil deep-frying oil, then did aerobic exercise, DFO+OCT group: rats were received deep-frying oil deep-frying oil, then were prescribed octopamine, DFO+EXE+OCT group: rats were received deep-frying oil deep-frying oil, then did aerobic exercise and were prescribed octopamine simultaneously. The results represent the mean $\pm S D$ with five repeats.

\section{Caspase3}

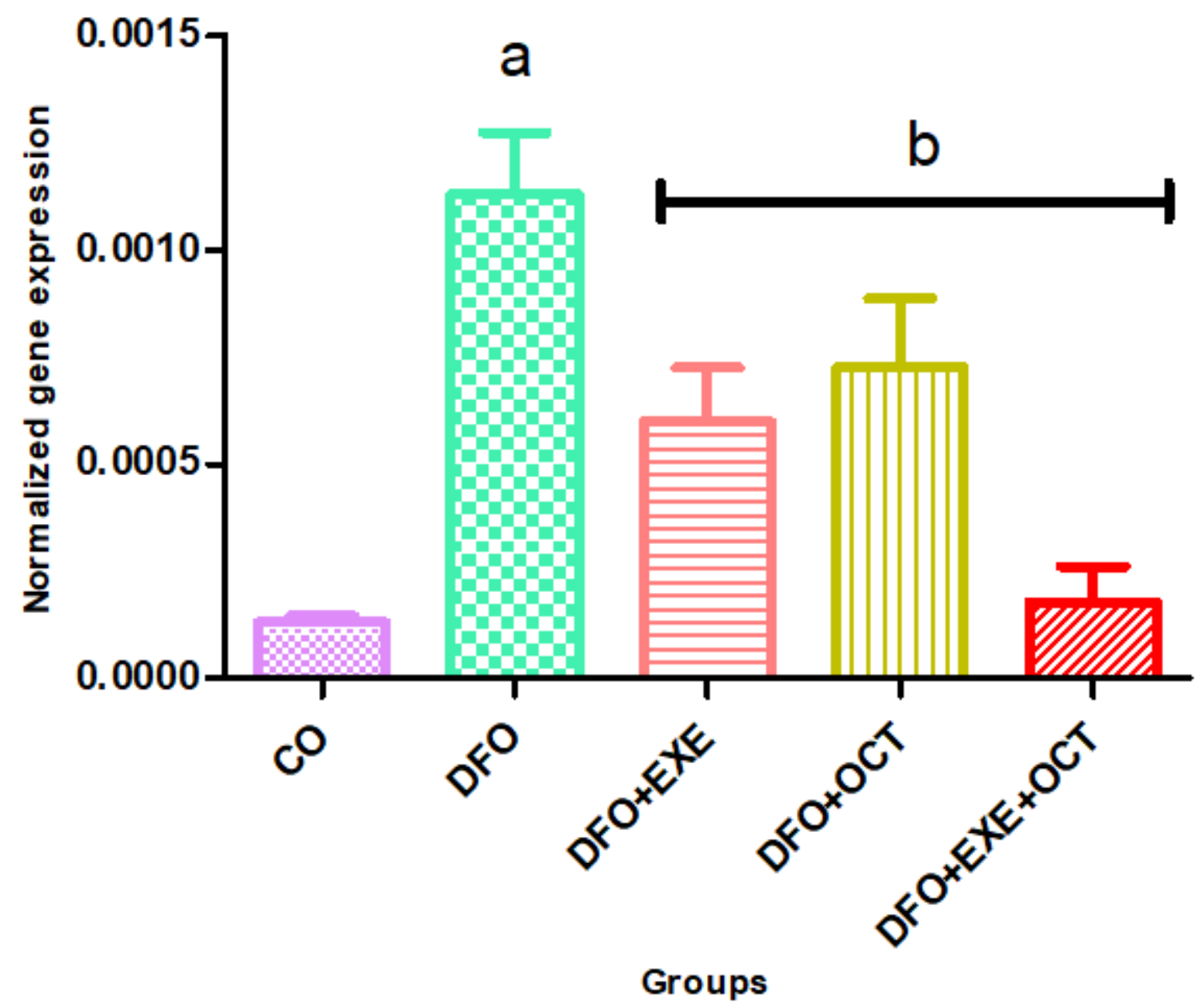

Figure 4

Average relative Caspase 3 expression in the group studied compared to GAPDH. a: significantly different from the control group (CO) at $P \otimes 0.002$, b: significantly different from the DFO at $P \otimes 0.01$. CO: control (rats were not received any treatments), DFO group: rats were received deep-frying oil, DFO+EXE group: rats were received deep-frying oil deep-frying oil, then did aerobic exercise, DFO+OCT group: rats were received deep-frying oil deep-frying oil, then were prescribed octopamine, DFO+EXE+OCT group: rats were received deep-frying oil deep-frying oil, then did aerobic exercise and were prescribed octopamine simultaneously. The results represent the mean \pm SD with five repeats. 

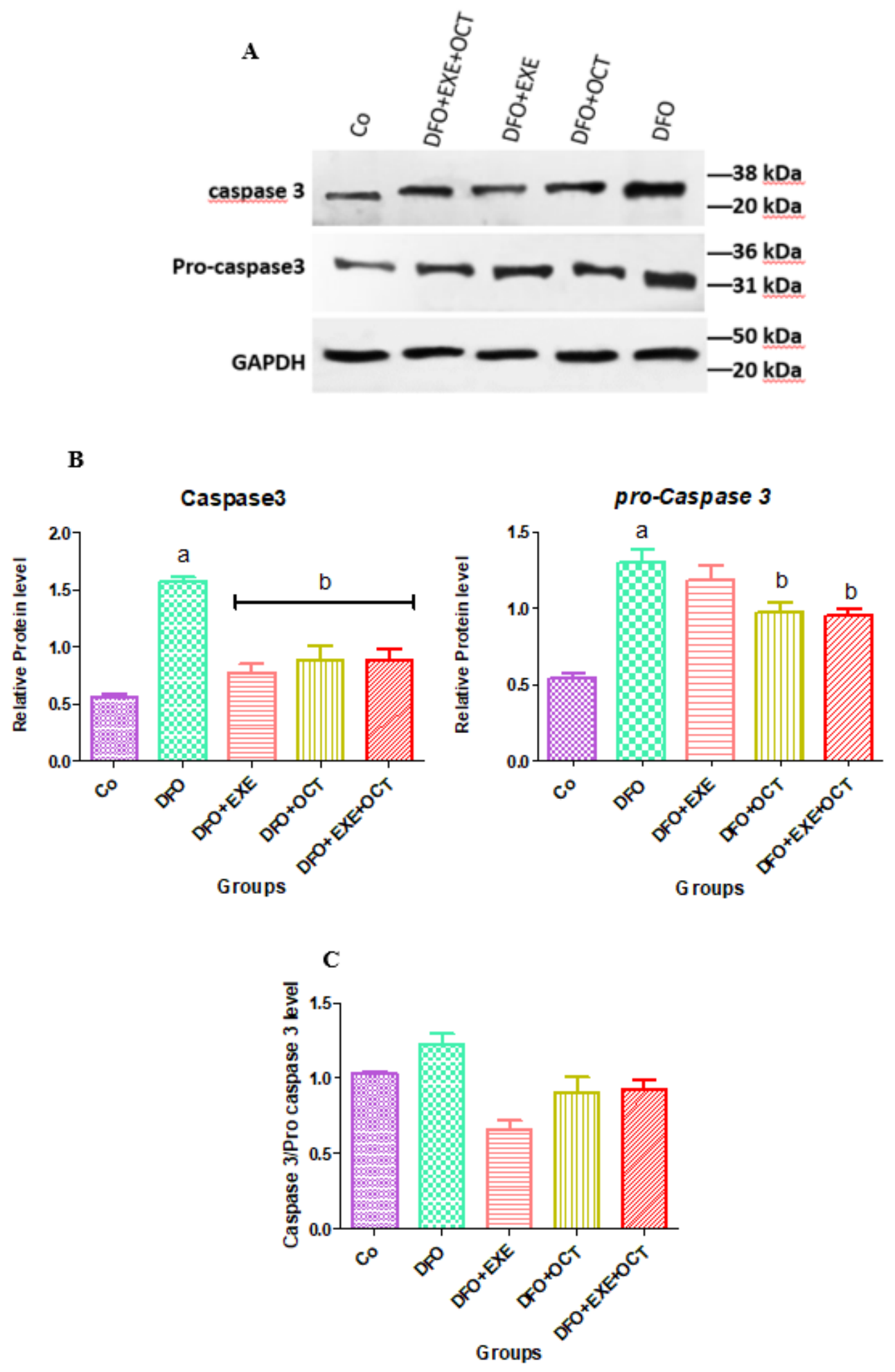

\section{Figure 5}

A) Western blotting analysis for Caspase 3 and pro-Caspase 3 proteins expression in different groups. GAPDH was used to confirm the normalization of the protein load. In western blotting bands, the groups are arranged in order of expression (low to high). B) Semi-quantitative analysis of western blot for different proteins. C) The ratio of activated caspase 3 to procaspase 3. a: significantly different with Co group at $P \otimes 0.01$, b: significantly different with the DFO group at $P \otimes 0.01, C 0$ : control (rats were not 
received any treatments), DFO group: rats were received deep-frying oil, DFO+EXE group: rats were received deep-frying oil deep-frying oil, then did aerobic exercise, DFO+OCT group: rats were received deep-frying oil deep-frying oil, then were prescribed octopamine, DFO+EXE+OCT group: rats were received deep-frying oil deep-frying oil, then did aerobic exercise and were prescribed octopamine simultaneously. The results represent mean $\pm S D$ with five repeats.

\section{Supplementary Files}

This is a list of supplementary files associated with this preprint. Click to download.

- Table1.docx

- 1485t.PDF 